\title{
Consumo prejudicial de álcool entre usuários da Estratégia Saúde da Família
}

\author{
Harmful alcohol consumption between users of Family Health Strategy \\ Ângela Maria Mendes Abreu', Rafael Tavares Jomar², Maria Helena do Nascimento Souza', \\ Raphael Mendonça Guimarães ${ }^{3}$
}

\section{PREZADO EDITOR,}

O consumo de álcool tem sido apontado como responsável por grande número de acidentes de trânsito, violência doméstica, aumento da morbimortalidade por doenças cardiovasculares, cirrose hepática e distúrbios psiquiátricos. Seu consumo abusivo está também relacionado a cerca de 2,5 milhões de mortes anuais em todo o mundo, sendo considerado o primeiro fator de risco para a carga global de doenças em países das Américas'.

Mais da metade da população brasileira consome álcool, 25\% dela já experimentou pelo menos um tipo de problema relacionado ao seu consumo, 3\% preenchem critérios para abuso e 9\% para dependência dessa substância². Portanto, estudos que identifiquem características sociais, demográficas e individuais associadas ao consumo prejudicial de álcool são necessários para o desenvolvimento de estratégias de redução desse padrão de consumo no Brasil³.

Apresentam-se aqui resultados preliminares de um inquérito domiciliar em andamento que objetiva identificar o consumo prejudicial de álcool entre residentes de áreas adscritas à Estratégia Saúde da Família (ESF) da cidade do Rio de Janeiro, Brasil. Para tanto, o Alcohol Use Disorders Identification Test (AUDIT), um dos instrumentos mais empregados no mundo para a identificação de grupos de risco e rastreamento do uso prejudicial de álcool que apresenta boa qualidade psicométrica no contexto brasileiro ${ }^{4}$, foi aplicado em amostra intencional de 196 usuários da ESF (144 mulheres e 52 homens), com idades entre 19 e 57 anos e idade média de 36,5 anos (+/-13,05).

A maioria dos entrevistados era casada (63,8\%), possuía ensino fundamental incompleto $(30,1 \%)$ e renda familiar mensal entre $1-2$ salários-mínimos $(55,6 \%)$. No tocante ao consumo prejudicial de álcool, 19,4\% dos entrevistados enquadraram-se nessa classificação. Nesse padrão de consumo, houve maior proporção estatisticamente significante (a um nível de 0,05 pelo teste $\left.x^{2}\right)$ entre indivíduos do sexo masculino $(p<0,001)$ e entre aqueles com renda familiar mensal superior a 3 salários-mínimos $(p<0,03)$.

Apesar de o presente estudo apresentar frequência maior, uma metanálise aponta que entre $6 \%$ e 15\% das pessoas que demandam cuidados em serviços de atenção primária à saúde fazem consumo prejudicial ou são dependentes de álcool, entretanto apenas um terço delas tem tal problema detectado pelos profissionais que as atendem ${ }^{5}$.

Avaliar sistematicamente o consumo de álcool, portanto, é importante para prevenir consequências sociais e de saúde nas populações, especialmente naquelas atendidas pela ESF 
que têm a promoção da saúde e a prevenção de agravos como práticas sanitárias prioritárias. Para tanto, acreditamos não somente na atuação dos profissionais da ESF na promoção de hábitos saudáveis diante do consumo de álcool, mas também na prática de intervenções eficazes de controle do consumo prejudicial dessa substância.

Apontam-se como limitações deste estudo a possibilidade de causalidade reversa, o fato de a amostra ser intencional e o elevado número de indivíduos do sexo feminino entrevistados (73,4\%), o que ocorreu pelo fato de a coleta de dados ter sido realizada somente no período diurno de dias úteis, fazendo com que o número de donas de casa entrevistadas fosse elevado, o que pode ter implicado ainda a elevada proporção de consumidores de baixo risco encontrada (80,6\%).

\section{REFERÊNCIAS}

1. World Health Organization. Global status report on alcohol and health. Geneva: World Health Organization; 2011.

2. Laranjeira R, Pinsky I, Sanches M, Zaleski M, Caetano R. Alcohol use patterns among Brazilian adults. Rev Bras Psiquiatr. 2010;32(3):231-41.

3. Silveira CM, Silveira CC, Silva JG, Silveira LM, Andrade AG, Andrade LHSG. Epidemiologia do beber pesado e beber pesado episódico no Brasil: uma revisão sistemática da literatura. Rev Psiq Clín. 2008;35(Supl 1):31-8.

4. Santos WS, Gouveia VV, Fernandes DP, Souza SSB, Grangeiro ASM. Alcohol Use Disorders Identification Test (AUDIT): explorando seus parâmetros psicométricos. J Bras Psquiatr. 2012;61(3):117-23.

5. Aertgeerts B, Buntinx F, Kester A. The value of the CAGE in screening for alcohol abuse and alcohol dependence in general clinics population: a diagnostic meta-analysis. J Clin Epidemiol. 2004;57(1):30-9. 